\title{
A Note on Common Names
}

The essays in this collection follow the American Ornithological Society's system for capitalization of common names of birds. For example, Hermit Thrush instead of hermit thrush; Snowy Owl instead of snowy owl. When not using the specific name, however, the term is lowercased (e.g., the thrush; an owl). Capitalization style used in previously published essays has been retained. 

When Birds Are Near 
